\title{
MODULES CLOSE TO SSP- AND SIP-MODULES
}

\author{
ABYZOV ADEL NAILEVICH, TRAN HOAI NGOC NHAN \\ AND TRUONG CONG QUYNH
}

\begin{abstract}
In this paper, we investigate some properties of SIP, SSP and CS-Rickart modules. We give equivalent conditions for SIP and SSP modules; establish connections between the class of semisimple artinian rings and the class of SIP rings. It shows that $R$ is a semisimple artinian ring if and only if $R_{R}$ is SIP and every right $R$-module has a SIP-cover. We also prove that $R$ is a semiregular ring and $J(R)=Z\left(R_{R}\right)$ if only if every finitely generated projective module is a SIP-CS module which is also a $C 2$ module.
\end{abstract}

\section{INTRODUCTION AND NOTATION}

Throughout this paper $R$ denotes an associative ring with identity, and modules will be unitary right $R$-modules. The Jacobson radical ideal in $R$ is denoted by $J(R)$. The notations $N \leq M, N \leq_{e} M, N \unlhd M$, or $N \subset_{d} M$ mean that $N$ is a submodule, an essential submodule, a fully invariant submodule, and a direct summand of $M$, respectively. We refer to [6], 9], [19, and [25] for all the undefined notions in this paper.

Recall that a module $M$ is called a SIP module (respectively, SSP module) if the intersection (or the sum) of any two direct summands of $M$ is also a direct summand of $M$ (see 12, 14, 24). It is known that every Rickart right $R$-module $M$ (i.e., every endomorphism of $M$ has the kernel a direct summand) has the SIP (see 17, Proposition 2.16]) and every d-Rickart right $R$-module $M$ (i.e., every endomorphism of $M$ has the image a direct summand) has the SSP ([18, Proposition 2.11]).

F. Karabacak and A. Tercan introduced the notion of SIP-CS module in [16]. A module $M$ is called an SIP-CS module if the intersection of any two direct summands of $M$ is essential in a direct summand of $M$. Note that SIP-CS modules are called SIPextending modules in [16]. It is known that every CS-Rickart module has the SIP-CS (see 2. Proposition 1.(4)]).

In this paper, we provide some characterizations of SIP, SSP, SIP-CS and CS-Rickart modules.

2010 Mathematics Subject Classification. 16D40, 16D80.

Key words and phrases. SSP-module, SIP-module, SIP-CS, CS-Rickarts. 


\section{SIP MODULES AND SSP MODULES}

Let $f: A \rightarrow B$ be a homomorphism. We denote by $\langle f\rangle$ the submodule of $A \oplus B$ as follows:

$$
\langle f\rangle=\{a+f(a) \mid a \in A\} .
$$

The following result is obvious and we can omit its proof.

Lemma 2.1. Let $M=X \oplus Y$ and $f: A \rightarrow Y$, a homomorphism with $A \leq X$. Then

(1) $A \oplus Y=\langle f\rangle \oplus Y$.

(2) $\operatorname{Ker}(f)=X \cap\langle f\rangle$.

We next study some properties of SIP and SSP modules via homomorphisms:

Proposition 2.2. The following conditions are equivalent for a module $M$ :

(1) $M$ is $S S P$.

(2) For any split monomorphism $f: A \rightarrow M$ with $A$ a direct summand of $M$, $A+\operatorname{Im}(f)$ is a direct summand of $M$.

(3) For any split epimorphism $f: M \rightarrow M / A$ with $A$ a direct summand of $M$, $A+\operatorname{Ker}(f)$ is a direct summand of $M$.

Proof. (1) $\Rightarrow(2),(3)$ are obvious.

$(2) \Rightarrow(1)$. Assume that $M=A_{1} \oplus A_{2}$ and $f: A_{1} \rightarrow A_{2}$ an $R$-homomorphism. Call $T=\langle f\rangle$ a submodule of $M$ and hence $M=T \oplus A_{2}$. We consider the homomorphism $\psi$ : $A_{1} \rightarrow M$ given by $\psi(x)=x+f(x)$. It is easily to see that $\psi$ is a split monomorphism. By (2), $A_{1}+\psi\left(A_{1}\right)=A_{1}+T$ is a direct summand of $M$. Furthermore, $A_{1}+T=A_{1} \oplus \operatorname{Im}(f)$, which implies $\operatorname{Im}(f)$ is a direct summand of $A_{2}$.

$(3) \Rightarrow(1)$. Suppose that $M=A_{1} \oplus A_{2}$ and $f: A_{1} \rightarrow A_{2}$ an $R$-homomorphism. Let $T=\langle f\rangle$ be a submodule of $M$. Then $M=T \oplus A_{2}$. Call the homomorphism $\psi: M \rightarrow M / T$ given by $\psi\left(a_{1}+a_{2}\right)=a_{2}+T$ for all $a_{1} \in A_{1}, a_{2} \in A_{2}$. Clearly, $\psi$ is a split epimorphism and $\operatorname{Ker}(\psi)=A_{1}$. By (3), $A_{1}+T$ is a direct summand of $M$. On the other hand, $A_{1}+T=A_{1} \oplus \operatorname{Im}(f)$, which implies $\operatorname{Im}(f)$ is a direct summand of $A_{2}$.

Corollary 2.3. The following conditions are equivalent for a module $M$ :

(1) $M$ is $S S P$.

(2) For any two direct summands $A_{1}$ and $A_{2}$ with $A_{1} \simeq A_{2}$, then $A_{1}+A_{2}$ is a direct summand of $M$.

Similarly with SIP, we also have some characterizations of SIP-modules:

Proposition 2.4. The following conditions are equivalent for a module $M$ :

(1) $M$ is SIP.

(2) For any split monomorphism $f: A \rightarrow M$ with $A$ a direct summand of $M$, $A \cap f(A)$ is a direct summand of $M$. 
(3) For any split epimorphism $f: M \rightarrow M / A$ with $A$ a direct summand of $M$, $A \cap \operatorname{Ker}(f)$ is a direct summand of $M$.

Corollary 2.5. The following conditions are equivalent for a module $M$ :

(1) $M$ is SIP.

(2) For any two direct summands $A_{1}$ and $A_{2}$ with $A_{1} \simeq A_{2}$, then $A_{1} \cap A_{2}$ is a direct summand of $M$.

Proposition 2.6. Let $R$ be a ring, $M$ an $R$-R-bimodule and $T=R \propto M$ the corresponding trivial extension. The following conditions are equivalent:

(1) T has the SSP

(2) (a) $R$ has the SSP.

(b) For every regular $x$ of $R$ with $x=x y x$, we have $x M(1-x y)=0$.

Proof. (1) $\Rightarrow(2)$. By [12, Proposition 4.5].

$(2) \Rightarrow(1)$. Assume that $x=x y x$. For any $m \in M$, call $z=x m(1-x y)$. It follows that $z=(x y) z(1-x y)$. Note that $x y$ is idempotent of $R$. By [12, Proposition 4.5], $z=(x y) z(1-x y)=0$.

Let $R$ be a ring and $\Omega$, a class of right $R$-modules which is closed under isomorphisms and summands. According to Enochs in [10], we study the notion of $\Omega$-envelope and the notion of $\Omega$-cover:

An $R$-homomorphism $g: M \rightarrow E$ is called an $\Omega$-envelope of a right $R$-module $M$; if $E \in \Omega$ such that any diagram:

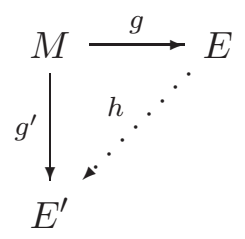

with $E^{\prime} \in \Omega$, can be completed, and the diagram:

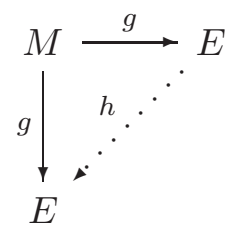

can be completed only by an automorphism $h$.

An $R$-homomorphism $g: E \rightarrow M$ is called an $\Omega$-cover of a right $R$-module $M$; if $E \in \Omega$ such that any diagram:

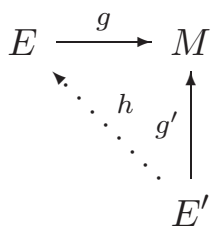


with $E^{\prime} \in \Omega$, can be completed, and the diagram:

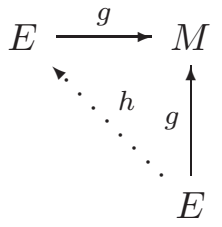

can be completed only by an automorphism $h$.

A right $R$-module $M$ is called a $C 3$-module if whenever $A$ and $B$ are direct summands of $M$ with $A \cap B=0$, then $A \oplus B$ is a direct summand of $M$. Dually, $M$ is called a D3-module if whenever $M_{1}$ and $M_{2}$ are direct summands of $M$ and $M=M_{1}+M_{2}$, then $M_{1} \cap M_{2}$ is a direct summand of $M$.

Proposition 2.7. The following conditions are equivalent for a ring $R$ :

(1) $R$ is a semisimple artinian ring.

(2) Every right $R$-module has a D3-cover.

(3) Every 2-generated right $R$-module has a D3-cover.

(4) Every right $R$-module has a D3-envelope.

(5) Every 2-generated right $R$-module has a D3-envelope.

Proof. (1) $\Rightarrow(2) \Rightarrow(3)$. Clear.

$(3) \Rightarrow(1)$. Let $S$ be a simple right $R$-module. Call $\varphi: R_{R} \rightarrow S$ an epimorphism. By (3), $M=R_{R} \oplus S$ has a D3-cover, say $\alpha: C \rightarrow M$ where $C$ is a D3-module. Let $\iota_{1}: S \rightarrow M$ and $\iota_{2}: R_{R} \rightarrow M$ be the inclusion maps for all $i=1,2$. Note that $S$ and $R_{R}$ are D3-modules, and there are homomorphisms $\beta_{1}: S \rightarrow C, \beta_{2}: R_{R} \rightarrow C$ such that $\alpha \beta_{i}=\iota_{i}$. Clearly, $i d_{M}=\iota_{1} \oplus \iota_{2}=\alpha\left(\beta_{1} \oplus \beta_{2}\right)$. This shows that $M$ is isomorphic to a direct summand of $C$, which implies that $M$ is a D3-module. We deduce that $\operatorname{Ker}(\varphi)$ is a direct summand of $R_{R}$ by [4, Proposition 4]. It follows that $S$ is a projective module. Thus $R$ is semisimple.

$(1) \Rightarrow(4) \Rightarrow(5)$. Clear.

$(5) \Rightarrow(1)$. Let $S$ be a simple right $R$-module. Call $\varphi: R_{R} \rightarrow S$ an epimorphism. By (5), $M=R_{R} \oplus S$ has a D3-envelope, named $\iota: M \rightarrow E$ where $E$ is a D3-module. Since $S$ and $R$ are D3-modules, there exist $f_{1}: E \rightarrow S, f_{2}: E \rightarrow R$ such that $f_{i} \iota=\pi_{i}$, where $\pi_{1}: M \rightarrow S$ and $\pi_{2}: M \rightarrow R$ are the projections. There exists $\phi: E \rightarrow M$ such that $\pi_{i} \phi=f_{i}$ for all $i=1,2$. It follows that $\phi \iota=i d_{M}$, and hence $\iota$ is a split monomorphism. Thus $N \oplus E(N)$ is isomorphic to a direct summand of $E$. This gives that $S \oplus R$ is also a D3-module. We deduce that $\operatorname{Ker}(\varphi)$ is a direct summand of $R_{R}$. So $S$ is a projective module. Thus $R$ is semisimple.

Corollary 2.8. The following conditions are equivalent for a ring $R$ :

(1) $R$ is a semisimple artinian ring.

(2) $R_{R}$ is $S I P$ and every right $R$-module has a SIP-cover.

(3) $R_{R}$ is $S I P$ and every 2-generated right $R$-module has a $S I P$-cover. 
(4) $R_{R}$ is SIP and every right $R$-module has a SIP-envelope.

(5) $R_{R}$ is SIP and every 2-generated right $R$-module has a SIP-envelope.

A ring $R$ is called a right $V$-ring if every simple right $R$-module is injective.

Proposition 2.9. The following conditions are equivalent for a ring $R$ :

(1) $R$ is a right $V$-ring.

(2) Every finitely cogenerated right $R$-module has a C3-envelope.

(3) Every finitely cogenerated right $R$-module has a C3-cover.

Proof. (1) $\Rightarrow(2),(3)$ are obvious.

$(2) \Rightarrow(1)$ Let $N$ be an arbitrary simple module. Assume that $\iota: M=N \oplus E(N) \rightarrow E$ is the C3-envelope, where $E$ is a C3-module. Since $N$ and $E(N)$ are C3-modules, there exist $f_{1}: E \rightarrow N, f_{2}: E \rightarrow E(N)$ such that $f_{i} \iota=\pi_{i}$, where $\pi_{1}: M \rightarrow N_{i}$ and $\pi_{2}: M \rightarrow E(N)$ are the projections. There exists $\phi: E \rightarrow M$ such that $\pi_{i} \phi=f_{i}$ for all $i=1,2$. It follows that $\phi \iota=i d_{M}$, and so the monomorphism $\iota$ splits. Thus $N \oplus E(N)$ is isomorphic to a direct summand of $E$. It follows that $N \oplus E(N)$ is also a C3-module. Therefore $N$ is a direct summand of $E(N)$. This gives $N$ is injective. Thus $R$ is a right V-ring.

$(3) \Rightarrow(1)$ The proof is similar to the proof $(3) \Rightarrow(1)$ of Proposition 2.7.

Similarly, we also get the following result for injectivity of semisimple modules:

Proposition 2.10. The following conditions are equivalent for a ring $R$ :

(1) $R$ is a right Noetherian right $V$-ring.

(2) Every right $R$-module with essential socle has a C3-envelope.

(3) Every right $R$-module with essential socle has a C3-cover.

\section{SIP-CS MODULES}

A module $M$ is called relatively CS-Rickart to $N$ (or $N$-CS-Rickart) if for every $\varphi \in \operatorname{End}_{R}(M, N), \operatorname{Ker} \varphi$ is an essential submodule of a direct summand of $M$. A module $M$ is called relatively $d$-CS-Rickart to $N$ (or $N$-d-CS-Rickart) if for every $\varphi \in$ $\operatorname{End}_{R}(N, M), \operatorname{Im} \varphi$ lies above a direct summand of $M$. A module $M$ is called CS-Rickart (d-CS-Rickart) if $M$ is $M$-CS-Rickart (resp., $M$-d-CS-Rickart). $M$ is called a SIP-CS module if $A_{i}$ is essential in a direct summand of $M$ for all $i \in \mathcal{I}, \mathcal{I}$ is a finite index set, then $\bigcap_{i \in \mathcal{I}} A_{i}$ is essential in a direct summand of $M$ (see [16]). $M$ is called a SSP-lifting module if $A_{i}$ lies above a direct summand of $M$ for all $i \in \mathcal{I}, \mathcal{I}$ is a finite index set, then $\sum_{i \in \mathcal{I}} A_{i}$ lies above a direct summand of $M$ (see [23]). The class of CS-Rickart (d-CS-Rickart, SIP-CS, SSP-lifting) modules is studied by the authors in [1, 2].

Lemma 3.1. The following implications hold for a module $M=M_{1} \oplus \ldots \oplus M_{n}$ :

(1) if $M$ is relatively $C S$-Rickart to $N$ then $M_{i}$ relatively CS-Rickart to $N$.

(2) if $M$ is relatively $d$-CS-Rickart to $N$ then $M_{i}$ relatively $d$-CS-Rickart to $N$. 
Proof. We only need to prove for the case $n=2, i=1$.

(1) Assume that $M=M_{1} \oplus M_{2}$ is relatively CS-Rickart to $N$. There exists $\varphi$ : $M \rightarrow N$ such that $\varphi=\left.\psi \oplus 0\right|_{M_{2}}$ for each $\psi: M_{1} \rightarrow N$. By assumption, there exists a direct summand $D$ of $M$ such that $\operatorname{Ker}(\varphi) \leq_{e} D$. Since $\operatorname{Ker}(\varphi)=\operatorname{Ker}(\psi) \oplus M_{2}$ and $D=\left(D \cap M_{1}\right) \oplus M_{2}$, it follows that $\operatorname{Ker}(\psi) \oplus M_{2} \leq_{e}\left(D \cap M_{1}\right) \oplus M_{2}$. Therefore $\operatorname{Ker}(\psi) \leq_{e} D \cap M_{1}$. Since $D$ is a direct summand of $M, D \cap M_{1}$ is a direct summand of $M_{1}$. Hence $M_{1}$ is relatively CS-Rickart to $N$.

(2) Assume that $M=M_{1} \oplus M_{2}$ is relatively d-CS-Rickart to $N$. There exists $\varphi: M \rightarrow N$ such that $\varphi=\left.\psi \oplus 0\right|_{M_{2}}$ for each $\psi: M_{1} \rightarrow N$. By assumption, $\operatorname{Im} \varphi=\operatorname{Im} \psi$ lies above a direct summand of $N$. Thus, $M_{1}$ is relatively d-CS-Rickart to $N$.

Proposition 3.2. The following implications hold for a module $M$ :

(1) if $M$ is a SIP-CS module with $C 2$ condition and $M=M_{1} \oplus M_{2}$ then $M_{1}$ relatively CS-Rickart to $M_{2}$.

(2) if $M$ is a SSP-lifting module with D2 condition and $M=M_{1} \oplus M_{2}$ then $M_{1}$ relatively $d$-CS-Rickart to $M_{2}$.

Proof. Let $f: M_{1} \rightarrow M_{2}$ be an $R$-homomorphism. Then $M=\langle f\rangle \oplus M_{2}$.

(1). We have that $\operatorname{Ker}(f)=\langle f\rangle \cap M_{1} \leq_{e} e M$ for some $e^{2}=e \in S$, by $M$ is SIP-CS. Let $\pi_{1}: M_{1} \oplus M_{2} \rightarrow M_{1}$ be the canonical projection and hence $e M \cap M_{2}=0$, implies that $\pi_{1}(e M) \cong e M$. Since $M$ is a $C 2$ module, $\pi_{1}(e M)$ is a direct summand of $M$. Then, since $\operatorname{Ker}(f) \leq_{e} e M, \operatorname{Ker}(f)=\pi_{1}(\operatorname{Ker}(f)) \leq_{e} \pi_{1}(e M)$. Hence, $M_{1}$ is relatively CS-Rickart to $M_{2}$.

(2). We have that $\operatorname{Im}(f) \oplus M_{1}=\langle f\rangle+M_{1}$ lies above $e M$ for some $e^{2}=e \in S$, by $M$ is SSP-lifting. Since $\langle f\rangle+M_{1}+M_{2}=M$, eM+ $M_{2}=M$ by [8, 3.2.(1)]. Let $\pi_{1}: M_{1} \oplus M_{2} \rightarrow M_{1}$ be the canonical projection. Then $\pi_{1 \mid e M}$ splits by $D 2$ condition. It follows that $e M=\left(e M \cap M_{2}\right) \oplus N$ by $\operatorname{Ker}\left(\pi_{1 \mid e M}\right)=e M \cap M_{2}$. We have $N \oplus M_{2}=$ $N+\left(e M \cap M_{2}+M_{2}\right)=e M+M_{2}=M$ and obtain that $N \oplus \operatorname{Im}(f)=M_{1} \oplus \operatorname{Im}(f) \supset e M$.

By modular law, $e M=N \oplus e M \cap \operatorname{Im}(f)$. As $\frac{\operatorname{Im}(f) \oplus M_{1}}{e M} \ll \frac{M}{e M}$, we have that $\frac{N \oplus \operatorname{Im}(f)}{N \oplus e M \cap \operatorname{Im}(f)} \ll \frac{N \oplus M_{2}}{N \oplus e M \cap \operatorname{Im}(f)}$. This is equivalent to $\frac{\operatorname{Im}(f)}{e M \cap \operatorname{Im}(f)} \ll$ $\frac{M_{2}}{e M \cap \operatorname{Im}(f)}$, which implies that $\operatorname{Im}(f)$ lies above the direct summand $e M \cap \operatorname{Im}(f)$ of $M$.

Corollary 3.3. The following implications hold for a module $M=M_{1} \oplus \ldots \oplus M_{n}$ :

(1) if $M$ is a SIP-CS module with $C 2$ condition then $M_{i}$ is relatively $C S$-Rickart to $M_{j}$ for every $i \neq j$.

(2) if $M$ is a SSP-lifting with D2 condition then $M_{i}$ is relatively $d$-CS-Rickart to $M_{j}$ for every $i \neq j$. 
Proof. If $M$ is a SIP-CS module with $C 2$ condition (respectively, SSP-lifting with D2 condition), then by Proposition [3.2, $\bigoplus_{i \neq j} M_{i}$ is relatively CS-Rickart to $M_{j}$ (respectively, relatively d-CS-Rickart to $M_{j}$ ). By Lemma 3.1, $M_{i}$ is relatively CS-Rickart to $M_{j}$ (respectively, relatively d-CS-Rickart to $M_{j}$ ) for every $i \neq j$.

Corollary 3.4. The following implications hold for a module $M$ :

(1) if $M \oplus M$ is a SIP-CS module with C2 condition then $M$ is a CS-Rickart module.

(2) if $M \oplus M$ is a SSP-lifting with D2 condition then $M$ is a d-CS-Rickart module.

Proof. Follow from Corollary 3.3 .

The singular submodule $Z(M)$ of a right $R$-module $M$ is defined as $Z(M)=\{m \in$ $M: a n n_{R}^{r}(m)$ is an essential right ideal of $\left.R\right\}$ where $a n n_{R}^{r}(m)$ denotes the right annihilator of $m$ in $R$. The singular submodule of $R_{R}$ is called the (right) singular ideal of the ring $R$ and is denoted by $Z\left(R_{R}\right)$. It is well known that $Z\left(R_{R}\right)$ is indeed an ideal of $R$.

Next we give a necessary and sufficient condition for a ring over which every finitely generated projective module to be a SIP-CS - module which is also a $C 2$ module.

Theorem 3.5. The following conditions are equivalent for a ring $R$ :

(1) $R$ is a semiregular ring and $J(R)=Z\left(R_{R}\right)$;

(2) Every finitely generated projective module is a CS-Rickart module which is also a $C 2$ module.

(3) Every finitely generated projective module is a SIP-CS module which is also a C2 module.

(4) Every finitely generated projective module is a SIP-CS module which is also a C3 module.

Proof. (1) $\Rightarrow(2)$. Follows from [2, Theorem 2].

$(2) \Rightarrow(3)$. Follows from [2, Proposition 1].

$(3) \Rightarrow(2)$. Let $P$ be a finitely generated projective module. By the hypothesis, $P$ is a SIP-CS module which is also a $C 2$ module. Then $P \oplus P$ is a SIP-CS module which is also a $C 2$ module. Since Proposition 3.2, $P$ is relatively CS-Rickart to $P$, it means that $P$ is a CS-Rickart module.

$(3) \Leftrightarrow(2)$. Follows from [1, Corollary 3.5].

Lemma 3.6. The following conditions are equivalent for a module $M$.

(1) $M$ is a SIP-CS module.

(2) Intersection of every pair of direct summands of $M$ is essential in a direct summand of $M$.

Proof. It is obvious.

Proposition 3.7. Assume that $M$ is a SIP-CS module. Then for any decomposition $M=M_{1} \oplus M_{2}$ and $f: M_{1} \rightarrow M_{2}$ is a homomorphism, then $\operatorname{Ker}(f)$ is essential in a direct summand of $M$. 
Proof. Assume that $M=M_{1} \oplus M_{2}$ and $f: M_{1} \rightarrow M_{2}$ an $R$-homomorphism. Call $T=\langle f\rangle$ a submodule of $M$. So $M=T \oplus M_{2}$ and $\operatorname{Ker}(f)=T \cap M_{1}$. On the other hand, by the hypothesis, $M$ is a SIP-CS and hence $\operatorname{Ker}(f)$ is essential in a direct summand of $M$ by Lemma 3.6 .

Corollary 3.8. Let $M$ be a module and $N$, a nonsingular module. If $M \oplus N$ is a SIP$C S$ module, then every homomorphism from $M$ to $N$ has the kernel a direct summand of $M$.

Proof. Let $f: M \rightarrow N$ be a non-zero homomorphism. By Proposition 3.7, $\operatorname{Ker}(f)$ is essential in a direct summand of $M \oplus N$. Assume that $A$ is a direct summand of $M \oplus N$ such that $\operatorname{Ker}(f) \leq_{e} A$. Call $\pi_{M}: M \oplus N \rightarrow M$ the canonical projection and $h=\left.\left(f \circ \pi_{M}\right)\right|_{A}: A \rightarrow N$. Therefore $\operatorname{Ker}(h)=\operatorname{Ker}(f) \oplus(N \cap A)$. We have that $\operatorname{Ker}(f) \leq_{e} A$ and obtain that $\operatorname{Ker}(f) \oplus(N \cap A) \leq_{e} A$. It follows that $\operatorname{Ker}(f) \oplus(N \cap A)=$ $A$. Thus $\operatorname{Ker}(f)$ is a direct summand of $M$.

Corollary 3.9. Let $M$ be an indecomposable module and $N$ be a nonsingular module. If $M \oplus N$ is a SIP-CS module, then every nonzero homomorphism from $M$ to $N$ is a monomorphism.

Proposition 3.10. Let $M$ be a nonsingular right $R$-module. If $(R \oplus M)_{R}$ is a SIP-CS module, then every cyclic submodule of $M$ is projective.

Proof. Let $m$ be a non-zero arbitrary element of $M$. Call the homomorphism $\varphi: R_{R} \rightarrow$ $M$ given by $\varphi(x)=m x$. As $(R \oplus M)_{R}$ is a SIP-CS module, $\operatorname{Ker}(\varphi)$ is a direct summand of $R_{R}$ by Corollary 3.8. It follows that $\operatorname{Im}(\varphi)$ is isomorphic to a direct summand of $R_{R}$. Thus $m R$ is a projective module.

A ring $R$ is called right (semi)hereditary if every (finitely generated) right ideal of $R$ is projective.

Theorem 3.11. The following statements are equivalent for a right nonsingular ring $R$.

(1) $R$ is right hereditary.

(2) Every projective right $R$-module is a SIP-CS module.

Proof. (1) $\Rightarrow(2)$ is obvious.

$(2) \Rightarrow(1)$ Let $I$ be a right ideal of $R$. We will show that $I$ is a projective module. Call an epimorphism $\varphi: F \rightarrow N$ for some free right $R$-module $F$. Let $\iota$ be the inclusion map from $I$ to $R_{R}$. Consider the homomorphism $\iota \circ \varphi: F \rightarrow R_{R}$. By (2), $F \oplus R_{R}$ is a SIP-CS module. We have from Corollary 3.8, $\operatorname{Ker}(\varphi)=\operatorname{Ker}(\iota \circ \varphi)$ is a direct summand $F$. This gives that $F=\operatorname{Ker}(\varphi) \oplus B$ for some submodule $B$ of $F$. Thus, $I$ is projective.

The author Warfied proved that if $R$ is right serial, then $R$ is right nonsingular if and only if $R$ is right semihereditary. 
The same argument of the proof of Theorem 3.11, we also have the following result of semihereditary rings:

Theorem 3.12. The following statements are equivalent for a right nonsingular ring $R$.

(1) $R$ is right semihereditary.

(2) Every finitely generated projective right $R$-module is a SIP-CS module.

(3) Every finitely generated free right $R$-module is a SIP-CS module.

Let $M$ be a right $R$-module and $S=\operatorname{End}(M)$. We denote

$$
\Delta(S)=\left\{f \in S \mid \operatorname{Ker}(f) \leq_{e} M\right\} .
$$

An $R$-module is called a self-generator if it generates all its submodules.

Theorem 3.13. The following conditions are equivalent for a self-generator module $M$ with $S=\operatorname{End}(M)$ :

(1) $S$ is a semiregular ring with $J(S)=\Delta(S)$.

(2) $M$ is a CS-Rickart and C2 module.

Proof. (1) $\Rightarrow(2)$ Assume that $S$ is a semiregular ring with $J(S)=\Delta(S)$. As $M$ is a self-generator, $J(S)=\Delta(S) \leq Z\left(S_{S}\right)$. We deduce that $S$ is right $\mathrm{C} 2$. This gives that $M$ is a C2-module by [21, Theorem 7.14(1)]. Let $\alpha: M \rightarrow M$ be an endomorphism of $M$. As $S$ is a semiregular ring, there exists $\beta \in S$ such that $\beta=\beta \alpha \beta$ and $\alpha-\alpha \beta \alpha \in J(S)$ by [20, Theorem 2.9]. Call $e=1-\beta \alpha$. Then $e^{2}=e \in S$. As $\alpha-\alpha \beta \alpha \in \Delta(S)$, $\operatorname{Ker}(\alpha-\alpha \beta \alpha) \leq_{e} M$ and hence $\operatorname{Ker}(\alpha-\alpha \beta \alpha) \cap e(P) \leq_{e} e(M)$. It is easily to check that $\operatorname{Ker}(\alpha-\alpha \beta \alpha) \cap e(M)=\operatorname{Ker}(\alpha)$. We deduce that $\operatorname{Ker}(\alpha) \leq_{e} e(M)$.

$(2) \Rightarrow(1)$ By [22, Theorem 3.2].

Corollary 3.14. The following conditions are equivalent for a self-generator module $M$ with $S=\operatorname{End}\left(M^{(\mathbb{N})}\right)$ :

(1) $S$ is a semiregular ring with $J(S)=\Delta(S)$.

(2) $M^{(\mathbb{N})}$ is a CS-Rickart and C2 module.

(3) $M^{(\mathbb{N})}$ is a SIP-CS and C2 module.

Proof. By Proposition 3.2 and Theorem 3.13 ,

\section{ACKNOWLEDGEMENTS}

The third author was supported by the Russian Government Program of Competitive Growth of Kazan Federal University. The author would like to thank the members of Department of Algebra and Mathematical Logic, Kazan (Volga Region) Federal University for their hospitality. 


\section{REFERENCES}

1. A. N. Abyzov, A. A. Tuganbaev, Modules in which sums or intersections of two direct summands are direct summands, Fundam. Prikl. Mat. 19:1 (2014) 3-11.

2. A. N. Abyzov, T. H. N. Nhan, CS-Rickart Modules, Lobachevskii Journal of Mathematics 35:4 (2014) 317-326.

3. M. Alkan, A. Harmanci, On Summand Sum and Summand Intersection Property of Modules, Turkish J. Math 26 (2002) 131-147.

4. I. Amin, Y. Ibrahim, M.F. Yousif, D3-modules, Comm. Algebra 42:2 (2014) 578-592.

5. I. Amin, Y. Ibrahim, M.F. Yousif, C3-modules, Algebra Colloq. 22 (2015) 655-670

6. F.W. Anderson, K. R. Fuller, Rings and Categories of Modules, Springer-Verlag, New York, 1974.

7. V. Camillo, Y. Ibrahim, M. Yousif, Y. Zhou, Simple-direct-injective modules, J. Algebra 420 (2014) 39-53.

8. J. Clark, C. Lomp, N. Vanaja, R. Wisbauer, Lifting Modules. Supplements and Projectivity in Module Theory, Frontiers in Mathematics, Birkhäuser, Basel-Boston-Berlin, 2006.

9. N. V. Dung, D. V. Huynh, P. F. Smith, R. Wisbauer, Extending modules, Pitman Research Notes in Math. 313, Longman, Harlow, New York, 1994.

10. E.E. Enochs, Injective and at covers, envelopes and resolvents, Israel J. Math. 39 (1981) 189-209.

11. C. Faith, Algebra II. Ring Theory, Springer-Verlag, New York, 1967.

12. J. L. Garcia, Properties of Direct Summands of Modules, Comm. Algebra 17:1 (1989) 73-92.

13. A. Hamdouni, A. Harmanci, A.Ç. Özcan, Characterization of modules and rings by the summand intersection property and the summand sum property, JP Jour.Algebra, Number Theory \& Appl. $5: 3$ (2005) 469-490.

14. J. Hausen, Modules with the Summand Intersection Property, Comm. Algebra 17:1 (1989) 135-148.

15. Y. Ibrahim, M. Tamer Kossan, T.C. Quynh, M. Yousif, Simple-direct-projective modules, Comm. Algebra 44 (2016) 5163-5178.

16. F. Karabacak, A. Tercan, On modules and matrix rings with SIP-extending, Taiwanese J. Math. 11 (2007) 1037-1044.

17. G. Lee, S. T. Rizvi, C. S. Roman, Rickart Modules, Comm. Algebra 38 (2010) 4005-4027.

18. G. Lee, S. T. Rizvi, C. S. Roman, Direct sums of Rickart modules, J. Algebra 353:1 (2012) 62-78.

19. S. H. Mohammed, B. J. Müller: Continous and Discrete Modules, London Math. Soc. LN 147: Cambridge Univ. Press., 1990.

20. W. K. Nicholson, Semiregular modules and rings, Canad. J. Math. 28 (1976) 1105-1120.

21. W. K. Nicholson, M. F. Yousif, Quasi-Frobenius Rings, Cambridge Univ. Press., 2003.

22. T. C. Quynh, M. T. Kosan, L. V. Thuyet, On (semi)regular morphisms, Comm. Algebra 41:8 (2013) 2933-2947.

23. Y. Talebi, A. R. M. Hamzekolaee, On SSP-lifting modules, East-West Journal of Mathematics 01 (2013) 1-7.

24. G. V. Wilson, Modules with the summand intersection property, Communications in Algebra 14:1 (1986) 21-38.

25. R. Wisbauer, Foundations of Module and Ring Theory, Gordon and Breach, Reading, 1991.

Department of Algebra and Mathematical logic, Kazan (Volga Region) Federal UniVersity, 18 Kremlyovskaya str., KazAn, 420008 Russia

E-mail address: aabyzov@ksu.ru, Adel.Abyzov@ksu.ru

Department of Mathematics, Danang University, 459 Ton Duc Thang, Danang city, VIETNAM

E-mail address: tcquynh@dce.udn.vn, tcquynh@live.com 
Department of Algebra and Mathematical Logic, Kazan (Volga Region) Federal UniVERsity, 18 KREMLYOVSKAyA STR., KAZAN, 420008 Russia

E-mail address: tranhoaingocnhan@gmail.com 